\title{
Architecture and the Protective Frame
}

\author{
Sally Augustin \\ Design With Science
}

\author{
Michael J. Apter \\ Apter International
}

\begin{abstract}
Architecture and interior design can have a significant effect on psychological state. Reversal theory helps to explain how and why design supports particular motivational and functional objectives - or fails to do so. Paratelic states require a protective frame; design can make it more likely that space-users in a paratelic state will have the desired experiences. Over the last fifty years, researchers in environmental psychology and related fields have identified sensory, psychosocial, and cultural factors that support confidence, safety-zone, and detachment protective frames as well as telic and paratelic states. Applying research findings in practice is complicated by the dynamic nature of people's motivations and, in some contexts, by the nuanced variety of human motivations among space users. Knowledge of reversal theory should help architects and others to deal more systematically and creatively with these kinds of problems and to enhance human experience.
\end{abstract}

Keywords: architecture, biophilic design, interior design, telic state, paratelic state, protective frame, reversal theory

Environments, natural and manmade, influence emotional state (for example, Russell \& Snodgrass, 1987). There are consistent patterns in how particular locations are experienced emotionally (for example, Wicker, 2012). Research has also specifically linked architecture, interior design, and psychological states experienced (Augustin, 2009). For example, in reversal theory terms, we might expect that the design of a church would normally play a part in inducing the serious and conforming states in worshippers, while the design of a spa would be intended to induce the sympathy and self-oriented states in clients.

One of the central ideas of reversal theory is that the paratelic state necessarily involves a protective frame and that this frame comes in many different forms, all of which protect the individual from danger or unacceptable negative consequences, at least as perceived by the individual. The aim of this paper is first to examine the part that architecture and interior design may play in inducing the paratelic state by means of the protective frame and the telic state by means of its absence. Secondly, it is to examine how architecture can support the needs of these two states once entered into. This paper can be seen as a continuation of both the reversal theory account of play (Kerr \& Apter, 1991), of what Apter has whimsically called "a theory of things" (Apter, 2014), and the use of the theory in design (Fokkinga \& Desmet, 2014a).

Correspondence concerning this article should be addressed to Sally Augustin, Principal, Design With Science, 535 North Ashland Avenue, La Grange Park, IL 60526. E-mail: sallyaugustin@designwithscience.com
Designers familiar with reversal theory have a deeper understanding of the human experience of space. They can use their knowledge of reversal theory to develop environments that more effectively support the full range of human motivations. Understanding reversal theory will also foster the design of spaces and objects that satisfy the fundamental human drives identified by reversal theory and do so in new and powerful ways. Reversal theory sensitizes the architect to the needs of the telic and paratelic states and provides insights about how they might be satisfied. This paper focus on telic and paratelic states; future papers will address the remaining six motivational states identified by Apter (conformist, negativistic, mastery, sympathy, autic, alloic).

\section{Three Kinds of Protective Frames}

At the core of the definition of the paratelic state is that it depends on the experience of a protective frame. The protective frame has been defined as a feature of experience in which the individual sees his or her situation as being basically safe from all serious negative consequences, be these physical, mental, or social. In this sense the protective frame encapsulates the experience and it means that actions are performed for their own sake rather than for their long-term consequences. In other words, the resulting paratelic state is essentially playful and play can be defined as action within this frame.

Reversal theory posits further that in the protective frame, arousal is experienced as pleasant excitement rather than unpleasant anxiety or anger. So when the protective frame is present, one wants to increase arousal as high as one can, and when it is absent, one wants to lower it as far as possible. 
Three kinds of protective frame have been suggested by Apter $(1991,2001,2006)$ and explored in the context of design by Fokkinga and Desmet (2014b).

\section{Confidence frame}

Here there is real danger, but the individual is confident that no harm will come, despite the threat. For example, looking at Niagara Falls, one experiences the threat of death that would occur on falling in, but this sight is pleasurably arousing because one is confident that the railing will in fact prevent one from falling. One is safe and then can enjoy the arousal that comes from the "danger."

\section{Safety-zone frame}

Here there is no real danger or at least danger is too distant to be concerned with. For example, one is several hundred feet away from the edge of the falls and therefore does not perceive any immediate danger. One is in a psychological zone of experienced safety. Within this zone, however, one welcomes anything that takes one's interest and causes arousal and this may include playful "danger."

\section{Detachment frame}

Here there is danger but it is observed and is experienced by others (fictional or real) not by oneself. In this sense one is not in real danger, even if one is aware of the "danger." One is merely a spectator or fantasist. For example, spectators are in the detachment frame while watching an action film in which one of the characters goes over the Niagara Falls.

\section{Designing protective frames}

What are the features of a building that allow it to act as a protective frame? In other words, what psychological mechanisms can be drawn on by an architect to use in inducing one or another of these three types of protective frame and the excitement that can come with them?

\section{Confidence Frame}

In this case the architect is faced with building something in a dangerous, or dangerous appearing, site but does so in a way that provides feelings of security to those using it (and spectators).

Examples of spaces that provide a confidence frame include:

- Fallingwater, built over a waterfall by Frank Lloyd Wright.

- Hitler's Eagles Nest, high up in Bavaria.

- Buildings (both ancient and modern) on the side of Mount Vesuvius.

- Buildings that seem subject to the threat of gravity, e.g. Tower of Pisa.
- Lighthouses, especially on dangerous promontories.

If you are only a spectator, then these are examples of the detachment frame. If, however, you own or inhabit such buildings, then these are indeed what we might call "confidence frame buildings."

For a confidence frame building to support a paratelic experience, it must seem solidly built and strong enough to withstand whatever the threat consists of. Without this, there is no frame and no playful state. Spaces with confidence frames support paratelic-type experiences because physical cues reassure individuals in these environments, as does their trust in the people who developed these spaces. For example, some materials, such as stone, traditionally linked by humans to strength and stability, make a space seem more comfortable (Kellert, 2008; Joye \& Verpooten, 2013).

Over time, the range of strength and trust evoking physical cues has expanded. In the Middle Ages, for example, a time of relatively frequent conflicts between local and national leaders, castles, whether they were perched in impregnable positions or on open planes, provided the people in them, gathered together for paratelic events such as festivals, with feelings of security from opportune enemy attacks via the apparent strength and thickness of their walls and stoutness of their beams.

In similar ways, astronauts in space can relax and enjoy mid-trip downtime because readily visible "strong" materials and sensors/gauges supplying information space users might utilize to save themselves, for example, make life-ending structural failure seem less likely in the inherently dangerous zone far above the Earth's surface.

People attending parties in embassies and other buildings around the world that might reasonably be expected to be attacked by terrorists have a better time because they've seen not only security guards during their visits to these high risk buildings, but also pylons to keep bomb-loaded cars from charging into these structures, courtyards in which troublemakers could be apprehended before they encounter guests, blast resistant materials, etc. Even if visitors to these environments can not effectively predict security measures that might be in place, most are easy to recognize when encountered and designers can enhance user experience by streamlining the process of identifying anti-terrorist measures, for example, by using traditional "security" materials such as steel, versions of familiar security forms such as gates, or signage indicating entrance to a secure area.

People eating dinner in the restaurants created in the most recent incarnation of the World Trade Center in New York City will be reassured by the well-publicized attempts to make the new structures on this site difficult to attack. There are massive steel fortifications at the base of these buildings, for example, and multiple news stories have covered these fortifications, which are largely invisible to the untrained eye once the building is completed. We may suppose that the ex- 
perience of diners in these areas may be enhanced with some feelings of "thrill." These applications of epoch-appropriate reassuring materials and process/systems are meaningless unless space users have reason to believe that they have been used/installed appropriately.

In spaces where armed attacks are less likely, for example, at viewing spots over natural features such as geothermal pools or special viewing decks on top of tall buildings or over chasms, designers have more freedom for how they communicate strength and stability and if they chose to directly do so or not. When materials used and design decisions don't straightforwardly and clearly convey strength, security, and trust, areas developed will only be used by individuals who are comfortable taking physical risks.

Spaces with confidence frames that are not vulnerable to attack are developed to communicate "safe danger;" they may have reinforced glass viewing areas, for example, from which visitors can look into the abyss, sometimes literally. A particularly spectacular example is the Grand Canyon Sky Walk that extends out nearly 4,000 feet over the floor of the Grand Canyon, a transparent horseshoe far above the valley floor. A special viewing station has been built on the 103rd floor of the Willis Tower (formerly known as the Sears Tower) in Chicago. It has a glass floor so people visiting it can look straight down at the ground, more than 1,300 feet below. When one of the layers of glass on this floor cracked, the situation was widely discussed in the local media. Statements by Willis Tower management indicting that the cracks were not a safety risk because of redundant safety systems seemed to be hesitantly received by press assigned to cover the incident. Ultimately, local celebrities visited the viewing area after repairs were made to restore confidence in the attraction. When structures are constructed using materials not generally linked to strength, faith in engineers/designers has more of an influence on feelings of safety. Again, we may assume that, once the security is experienced as real, people will enjoy their visits and the added excitement that has been created for them.

In some earthquake prone parts of the world, visiting bars and restaurants at the top of tall buildings can be a paratelic experience. People in these environments avoid an anxious state and make a paratelic one more likely by considering building codes that make building collapse unlikely and by looking for indications that the structure is safe. For example, although people in tall buildings in earthquake zones expect to experience some building sway, movement that individuals perceive as excessive, with comparisons based on their prior experiences, may undermine feelings that the building is a safe place to be. In this situation, it seems more likely that a paratelic state will be converted into an anxious one than that the swaying of the building will pleasantly increase arousal levels.
To generalize the concept of confidence frame beyond safe buildings, here are some very different examples. First of all, think of being on a cruise ship far from shore and therefore far from ultimate safety if there is a storm. Any arousal will be likely to be felt as excitement if the ship and cruise provide sufficient feelings of confidence. For example, confidence is likely to ensue if life rafts and safety apparatus are visible, there are safety drills, and the crew has crisp neat uniforms, which communicate that they are trained and competent. A second example is the use of food trucks. These have traditionally been associated with food that would generally be considered marginally acceptable. But since many of these trucks began using new and eye-catching designs, people are more willing to take a risk with the safety of the food. Indeed, the experience might be enhanced because it is a little questionable and unpredictable. Thirdly, in some natural situations, such as people surfing in huge waves just before hurricanes strike, no built environments provide security, but reliance on personal ability and availability of help (fire and police squads) if all does not go well do provide a sufficient confidence frame for surfers to continue their risky behavior.

There are also, of course, buildings designed specifically to produce excitement: roller coasters, fun fair rides, towers, London's Eye, etc. They have to seem dangerous, but they are not really so; otherwise, they would attract few paying customers. Here we see the same principles, but writ large.

\section{Safety-Zones Frame}

The experience of a situation as being in a safety-zone can be enhanced in a number of ways architecturally to emphasize the distance from danger. Unlike the confidence frame, which involves starting from danger and then adding safety, in the safety-zone one starts from safety and then adds danger - or some other form of stimulation - to produce pleasant arousal.

As with the confidence frames, feeling secure enough in a safety-zone frame so that paratelic experience ensues can require particular behaviors on the part of others in a space. For example, in a restaurant that has been designed with a punk décor, developed to make it seem that punk subculture type events might occur in the space, waiters must interact with diners in a way that's consistent with that decorative style or an element of danger will not be introduced to the space. Cues tied to an assortment of sub-cultures can be used to add a feeling of danger to a space. The extent to which designers can rely on stereotypical design elements when creating these spaces depends on the extent to which people who will use a space are truly familiar with the culture being represented or are just dipping into it for a few days or a meal.

Several years ago, graffiti art became a popular way to add "an edge" to a space. Graffiti has traditionally been found in relatively "tough" neighborhoods and its use may evolve a 
space from boring to "worth a visit" status, at least among connoisseurs of the art of graffiti.

Designers can create safety-zone frames by recreating spaces that would be dangerous in the natural world inside built environments. For example, views into atriums from a few floors up are analogous to being on the edge of a cliff. An exuberant fountain brings turbulent rivers to mind, at least deep in our subconscious. Containment prevents these design elements from seeming truly dangerous - atrium overlooks have protective railings and turbulent fountain water is contained within concrete or tiled or otherwise well-defined spaces. Users must believe railings are properly installed, however, for paratelic experiences to result.

Designers can also take a natural experience to an extreme and couple it with a support program (i.e., trained personnel to support positive user experiences) and generate a paratelic event. For example, Ice Hotels in Sweden, Quebec, Finland, Japan, Norway, and elsewhere are constructed each winter entirely from blocks of ice. In these hotels, experiences common to conventional hotels, such as sleeping, are given an aura of danger. This space is purpose built to provide people staying in it with a special thrill, but is co-located with a more traditional hotel. The experience of staying in the hotel is exhilarating and not terrifying because support staff is trained to prevent calamities and modern and traditional tools (for example, sophisticated pads under sleepers that prevent them from freezing to death during the night and fur coats that guests may bring onsite) combine to keep danger at bay.

Later, we review elements of spaces that increase their arousal level. Using many energizing features in a space will create such a high-energy feeling that people in the environment will become hyper-alert, which can intensify the experience of fundamentally positive situations and create much the same effect as a safety frame. A colorful carnival-type environment complete with energizing surface and light colors; loud, fast-paced music, and stimulating scents would create this effect if the stimuli involved, such as scents and sounds, have positive associations.

Many places with a safety-zone frame have been designed to be completely enclosed, with no windows to the outside world. Since at least Elizabethan times (see the Globe theatre in London) most theatres have been completely cut off physically from the outside world. Casinos, "big box" stores, sports bars, museums, art galleries, concert halls, sports stadia (especially football stadia like the Superdome), the Coliseum in Rome. These all turn inwards and in some sense deny the existence of the external world, which can distract people in the space from the actions desired by those who manage the location. Then, having induced the playful state, these facilities do things to produce excitement within the state they have helped to create. Such things include playing competitive sport, gambling, listening to or watching narratives unfolding, getting challenged or surprised, etc.
This can be to the disadvantage of the individual who believes that everything is cut off from the real world and who therefore might take risks that are inappropriate. Thus the casino (with no windows and plastic "money") tells the individual that there are not real consequences for losing — but of course there are (Apter, 2007).

The safest place in the world is home and people naturally turn to home when they are in danger. In developing their homes, people often do the opposite of what architects want or expect them to do. They buy ornaments and knick-knacks, they use cupboards for storing things that do not really fit, and so on. But in doing so they make a building into a personal and individualized home. Familiarity in hotel rooms - developed by designing the décor the same in every hotel in the chain - can make them more comforting and comfortable spaces to stay. Familiarity increases comfort with a space (De Vries, Holland, Chenier, Starr, \& Winkelman, 2010), which supports telic motivations.

\section{Detachment Frame}

The detachment frame allows the individual the opportunity to identify with something outside hisself or herself and then enjoy their struggles as if they were themselves the ones struggling. The cinema, the boxing booth, the video game, the theatre - these are all examples. Home theatres, which isolate users from scenes viewed, just as full scale theatres do, are becoming increasingly popular in American homes as movie viewing and video game playing are becoming more and more prevalent, culture-wide. In all these cases, attention is directed to some common focus for identification. In an amphitheater, most clearly, the architect points you to a common source of interest. Sometimes this focusing can be achieved through a screen (cinema), which after all seems like a hole to a new reality. Or there can be a window with a beautiful scene (looking out over a beach, for example). Any kind of virtual reality would come under this heading, too, provided it is clear that it is virtual.

To summarize: In the confidence frame, the architect starts with danger and then adds safety. In the safety-zone case the architect starts with safety and then adds in something arousing and this may be a form of danger. Either of these may apply in the detachment case, the difference from the other two being that the observer is not physically present in relation to the danger, which is either seen from a distance or is imagined.

Any particular building may represent more than one of these three types of frame. For example, the theatre is both in the safety zone for most people and functions through detachment.

\section{Motivational State and Physical Environment}

Given that environments can help to induce telic or paratelic states through protective frames or their removal, 
what can we say about how the environment impacts the behavior that takes place in it once the individual has adopted the telic or paratelic state? What has research shown that could be used by the architect to enhance or optimize the effectiveness of the behavior involved?

We need to remark that of course many effects of environment on psychological state will be the same whether the ongoing state is telic or paratelic. For example, the colors used in a space significantly affect the perceived temperature there, with cool colors reducing the apparent temperature and warm colors increasing it (Fehrman \& Fehrman, 2000). So, painting an entryway blue makes people feel a little cooler when they enter a home than they would if the same space was painted a warm color. Wall color can therefore have environmental implications; it allows thermostats to be set to more earth friendly levels without compromising user experience. This is obviously true whether one is in the telic or paratelic state.

Research has established a number of ways that design can influence arousal levels. For example, colors that are not very saturated but relatively bright are calming to look at while the reverse is true of colors that are saturated but not very bright (Valdez \& Mehrabian, 1994). Similarly, more complex or incongruous visual patterns increase the energy levels of viewers (Mahnke, 1996). Another example: seeing wood grain is deeply calming (Fell, 2010).

The environmental impacts that are most relevant here are ones that relate to the telic and paratelic states and to the motivations and emotions that relate to them differentially. Reversal theory introduces distinctions that help us to think in a more sophisticated way about how the physical environment influences experience. For example, the experience of living on Earth makes humans expect that darker colors will be on lower surfaces and lighter colors on higher ones; other color placements make us more energized and aroused when we see them (Durão, 2007). This information is interesting, in general, but to use it in conjunction with reversal theory we need to know that when darker colors aren't lower the energized state is tense and uncomfortable. Likewise Knoop (2007) recommends using cool lights for alertness and warmer lights for relaxation, but what would we use for boredom and/or anxiety? We are told that under a bright light we are more likely to be in a positive mood (Knez \& Enmarker, 1998) - and to use reversal theory we need to know that that mood is relaxation. We learn that listening to nature sounds, including that of the ocean, has been linked to lower stress levels Schweitzer, Gilpin, \& Frampton, 2004). Reversal theory practitioners understand that the type of stress reduced is anxiety stress (telic).

Additional data, if we are allowed to make reasonable inferences, does tell us something, which could be used in reversal theory-informed design. For example, a silent environment (which is difficult to achieve) is just as stressful as one that is perceived to be too loud (Schweitzer, Gilpin, \& Frampton, 2004). In this case it seems reasonable to suppose that the silent environment will be more stressful to the individual in the paratelic state and that noisy environment to the person who is at that moment telic. Dazkir \& Read (2012) had people look at images of rooms containing either primarily rectilinear or primarily curvilinear furniture. As the researchers reported, "the curvilinear settings elicited higher amounts of pleasant-unarousing emotions, (such as feeling relaxed, peaceful and calm) than the rectilinear settings." Since we know that low arousal is more pleasant in the telic state, we can infer that curvilinear settings were indeed enjoyed more in the telic state.

Biophilic design, which is applying the same design tenets in built spaces that can be discerned in naturally occurring outdoor spaces where humans are, and have felt, at ease, undergirds many current place design efforts. Spaces that are biophilicly designed are psychological comfortable environments for humans (Kellert, 2012). That means that in biophilicly designed spaces the telic mindset is definitely supported; violation of biophilic design principles can increase the arousal levels of people.

As outlined in Kellert, Heerwagen, and Mador (2008), biophilicly designed spaces share certain "design principles" with the sort of environments where our species felt comfortable in its early years. In biophilicly designed environments people feel secure and can easily review the world around themselves, as someone sitting in a tree or in a cave with a view out over a valley does. Biophilicly designed spaces feature long sight lines, but these views can be through openings such as doorways; biophilicly designed spaces can have subdividing walls. Air moves gently in biophilicly designed spaces, as a result of a breeze from an open window or an air current created by an HVAC (heating, ventilation, and air conditioning) system. Natural light and materials, as well as plants, are significant elements of biophilicly-designed spaces that invite exploration at multiple levels. As we look at moss covered ground, we find pebbles that intrigue us, for example, and similar experiences can be built into designed environments. Our experiences change from hour-to-hour, day-to-day, and season-to-season in biophilicly-designed environments, just as there are seasonal variations in any natural environment. As biophilic design elements are eliminated, a space becomes more suitable for paratelic activities because arousal levels increase, eventually becoming too high for the successful completion of telic tasks. For example, as long sight lines are eliminated by walls, experienced mood changes.

We must also remember that to optimize experience, psychosocial issues such as having privacy when desired, appropriate personal space, territories (for individuals and groups), control over physical experiences, and culturally consistent 
design need to be recognized when spaces are being developed.

There is evidence that people prefer sensory experiences consistent with their motivational state, more relaxing experiences while they're in telic ones and more energizing ones when they're in a paratelic state (Walters, Apter, \& Svebak, 1982). In this study, results "supported the reversal theory thesis that low arousal [sensory] preference is associated with seriousness and planning orientation (all these characterizing the 'telic state') and that high arousal preference is associated with playfulness and spontaneity (all these characterizing the 'paratelic state')."

It should be recognized that there are place type variations that make particular sorts of sensory and psychosocial experiences more or less desirable. For example, when people are doing utilitarian shopping, an environment that supports telic motives is most desirable, but when they are shopping hedonically, a paratelic one is optimal. Also, different people in the same space may have varying objectives. For example, some people sitting in café tables in a particular section of a student center may be trying to study, while other students, sitting a few feet away, may be socializing. Practical reasons as well as individual differences may cause people to perceive and pursue conflicting behaviors in the same space. The café space noted may be the only area where students can eat on campus between meals, for example, so people who have missed meals but need to study may have temporarily relocated to the same set of tables being used by people celebrating a birthday.

\section{Behavior Settings and Related Concepts}

For several decades, environmental psychologists have been investigating links between the physical parameters of the settings that people experience and their thoughts and behaviors. Many of their efforts have paralleled those of researchers developing reversal theory, although reversal theory is not well known among environmental psychologists.

Architecture and interior design make particular mental states more likely. They effectively influence the mood of people experiencing them. As Russell and Snodgrass (1987) report, "the most important fact about emotional links to places may be their pervasiveness... Affective quality is the bottom line of an accounting of the many features in a place and is, we believe, a guide for much of your subsequent relationship to that place-what to do there, how well it is done, how soon to leave, whether or not to return." In addition, perceptions arising from the experience of being in a space are more significant than objectively assessed events, "Behavior may be influenced by the (estimated, perceived, or remembered) affective quality of an environment rather than by its objective properties directly" (Russell \& Snodgrass, 1987).

Russell and Lanius (1984) presented an effective and efficient method for categorizing affective qualities of environ- ments, which can be used to describe the emotional experience of natural and designed spaces and objects (1984). Two axes are used, one that ranges from pleasant to unpleasant and a second, perpendicular on that runs from calming to energizing. Spaces that are pleasant and energizing might be described as exhilarating, interesting, or exciting, for example. Those that are pleasant but calming can be categorized as peaceful, tranquil, and serene, while calming but unpleasant spaces are boring, dreary, and monotonous. Finally, spaces that are unpleasant but energizing are frenzied, hectic, and panicky. Those familiar with reversal theory will immediately recognize that this is essentially the same scheme as the one proposed by Apter (1982), which predates the work of Russell and Lanius and adds to it that one axis represents the telic state and the other the paratelic state. Russell and Lanius are cited here because of their impact specifically on environmental psychology.

Environmental psychologists, including Russell and Snodgrass, have indicated the importance of aligning the space-in-use with intended activities. As Russell and Sondgrass describe, "The preferred level of arousal also varies with the task to be performed: Given a difficult or unpleasant task subjects preferred a less arousing environment; given a simple or pleasant task, subjects preferred a more arousing environment" (Russell \& Mehrabian, 1975, 1978; Russell \& Snodgrass, 1987). This goes back to the classic work of Yerkes and Dodson (1908), giving rise to the Yerkes-Dodson Law. Here, reversal theory makes another distinction, which is between the hedonic tone of different levels of arousal and the performance attributes of different levels of arousal. Thus one may be in the telic state experiencing high arousal as anxiety, but be willing to tolerate this in order to better achieve important goals. This kind of choice needs to be taken into account by designers.

Furthermore (note that "plan" here means intention, not necessarily a long-term plan in the telic sense):

the single most important thing that a person brings to an encounter with the environment is the plan. Presumably the place was chosen to maximize success of the plan and presumably the place will be viewed positively if it does so... the single most important environmental variable affecting mood and affective appraisal may be the environment's ability to fulfill the goal. .. the physical environment can hinder our plans in many ways, with results ranging from slight deviations in the sequence of activities to large-scale disasters (Russell \& Snodgrass, 1987).

The experience of particular psychological states has thus clearly been established as a fundamental parameter of a setting. The scales along which these states can be described 
are also clear. As detailed in the next few paragraphs, the particular psychological states experienced can be linked to expected scenarios in particular locations as well as sensory and psychosocial factors.

The concept of "behavior settings" was introduced more than five decades ago by early environmental psychologists. Behavior settings are the holistic combination of a behavioral script and a particular location. Behavior is predictable and regular in many behavior settings and members of a culture learn the "lines" for a certain spot; particular behaviors are expected in these places (Barker, 1968). For example, people raised several decades ago expect to sit quietly and read or engage in similar activities in an academic library (although younger generations may be learning different scripts for these spaces) and to gather inpatient-focused conversations in hospital rooms. Misalignments between a person's intentions/activities and a behavior setting program lead to stress. Behavior settings can thus align, or not, with telic and paratelic motivations. Some locations have several different behavior setting programs and the one to apply would be clear to people familiar with the culture of the space developers. For example, a public space (outside the sanctuary) in a church might be used for prayer meetings, bingo games, and wedding receptions at different times, with each use linked to specific behavior setting scripts and potentially different states (i.e., telic or paratelic). We should not "chronotype" places and spaces any more than we do people.

Researchers studying behavior settings have recognized the emotional experiences of people in a particular place only as they are derived from consistencies between a space's script and the intended activities and sense-making of people in that environment (Wicker, 2012). As Wicker details in his 2012 work,

Applied to people's encounters with behavior settings, experience includes what occupants sense or feel from direct sensory contact with physical stimuli and happenings in settings and their adjustments to them. It also includes the residuals of such contact... in any given setting, different occupants may achieve different patterns of satisfactions and settings of different setting genotypes provide different satisfaction possibilities (e.g., Barker, 1987). However, experience in settings is not limited to immediate sensory contact and its residuals. It also includes symbolic meanings that occupants attach to activities, objects, and spaces that they encounter (see Mazumdar, 1992, 2009). For example, power, authority, and exclusivity may be sensed in offices that occupy larger spaces, are located on higher floors, are furnished more elegantly and have procedures and layouts that restrict visitor access. Religious worship services typically incorporate a variety of artifacts, architectural features, and rituals that are rich in symbolic meaning and that may contribute to feelings of awe, inspiration, and community.

The differences between telic and paratelic behavior setting scripts are related to society's general expectations for action in particular places as well as the sensory and psychosocial experiences we have in certain environments. For example, an in-library academic lecture hall (telic space) is filled with seats lined up in rows, making eye contact difficult. In the break room for librarians in the same building, where staff members will socialize, seats are arranged around circular tables and in other ways that facilitate easy eye contact, which aligns with more social, paratelic behavior. Behavior settings can include a protective frame or not, although protective frames must be present for paratelic states to be experienced. The physical embodiment of a frame may or may not be present in the paratelic state. It is important to note that people in the same physical place can be in different states - exam proctor vs. people taking exam, for example although all can be expected to be familiar with the behavior setting script for an environment.

The programs of behavior settings can align not only with paratelic and telic states, but also the other states that undergird reversal theory (i.e., conformist, negativistic, mastery, sympathy, autic, and alloic).

Kerr and Tacon (1999) presented study participants with four different locations and judged their responses to them. Consistent with work done on behavior settings, they report "significant differences between the four presentations on serious-playful, planning-spontaneous, felt arousal, and effort items... These results suggest that individuals are sensitive to the psychological states required in particular settings." An assortment of environments were investigated: a university library (telic), a lecture theatre just before a statistics lecture (telic), a sports center (paratelic), and a party in the student union (paratelic).

Kerr, Hayashi, Matsumoto, and Miyamoto (2002) built on Kerr and Tacon's earlier work by investigating metamotivational state and level of arousal in several settings. "The first setting (telic/low arousal) was a university medical library where participants were revising... the second setting (paratelic/low arousal) was a popular beach where people sunbathing were approached, the third setting (paratelic/high arousal) was at an amusement park just after participants rode on a roller coaster, and the fourth setting (telic/high arousal) was an indoor sports facility 15 minutes before the participants performed in an important sports meet." These researchers confirmed the earlier findings by Kerr and Tacon (1999) that "different metamotivational states and arousal levels are affected by different settings... it seems likely that individuals used at least two of the settings... (sunbathing at the beach and riding on a roller coaster) as part of their 
deliberate self-regulation of state and arousal... [and also confirmed] that changes in environmental events could induce reversal." This work by Kerr, Hayashi, Matsumoto and Miyamoto is also important because it indicates that placebased elements of reversal theory are consistent in Western and in non-Western cultures, their data being collected in Japan.

Physical experiences, designed and otherwise, influence arousal levels of individuals and groups; some are clearly more relaxing while others are more energizing. Sensory and psychosocial experiences can support protective frames by influencing their apparent efficacy and mood, in general. Sensory and psychosocial experiences can also support behavior setting scripts or make them more difficult to pursue. For example, if the surface and light colors used in an academic library are highly arousing, it is more difficult for people to achieve telic objectives there, although these colors do not necessarily reverse users into the paratelic state. It is important to differentiate lowered arousal levels, i.e., relaxation, which is pleasant in the telic state and the removal of threats (i.e., the strengthening of protective frames), which are consistent with increased pleasant arousal in paratelic ones.

\section{Destroying the Protective Frame}

What would an architect do if he or she wanted to destroy the protective frame, or build something that was designed to incorporate features that would do this and therefore induce and support the serious telic? By "destroy" here we do not of course mean anything permanent, we mean just causing active states to reverse, i.e., in this case, the telic temporarily replacing the paratelic.

While physical design influences thoughts and behaviors in context with features of the social and cultural context, it cannot determine them except in extreme conditions. Although Norman does not mention reversal theory, he does indicate experiences significant enough to immediately throw people into a strong negative mood, removing them from a paratelic state (2004). Examples of these experiences include: "sudden, unexpected loud sounds or bright lights; 'looming' objects (objects that appear to be about to hit the observer); extreme hot or cold... extremely bright lights or loud sounds... harsh, abrupt sounds; grating and discordant sounds."

Buildings that are visually sterile, cold and over-clean, white and shiny, might all have this function of destroying the protective frame, as we see in law courts, some churches, hospitals, police stations. Buildings with no comforts, no chairs or benches, in particular, can seem threatening. Ironically, some opposite features seem also to destroy feelings of being protected: dark buildings that look grimy and express seemingly medieval characteristics.

This destruction of the protective frame is often done inadvertently. For example, the diagrams of diseases and bod- ily organs that are prone to be used to decorate the cubicles where the patient waits for the doctor, are perfect to induce the telic state. More subtly, one thinks of the piles of fashion and golf magazines in dentist's waiting rooms.

If a high-arousal situation is created involving dangersignaling stimuli an unpleasant situation results. For example, at the Holocaust museum in Washington, DC, the space is packed with discomforting stimuli and the overall effect of being in the museum is extremely unpleasant, by design. For example, humans feel more comfortable in spaces where more lines are curvy than rectilinear, although in no successful space are all lines either rectilinear or curvilinear (Dazkir \& Read, 2012). Rectilinear lines predominate in the museum. In addition, some support structures overhead in the Holocaust museum look like downward focused triangles and these shapes above human heads have been linked to negative stress. Visitors to the museum also notice a strong, but difficult to identify odor. Materials about the museum available to visitors identify it as the smell of leather, specifically, of leather shoes. One display in the museum features leather shoes removed by people entering crematoria at concentration camps. Thus, the designers of the structure and program at the Holocaust museum intentionally use a variety of sensory experiences to create an arousing and unpleasant experience for visitors. The experience does not reverse people into a paratelic state, as most museums do, because it deals with extremely serious and upsetting matters, although visitors to the museum do learn many things about the Holocaust, many in a deeply emotional way and this may provide its own telic satisfactions.

People designing the National September 11 Memorial Museum at the World Trade Center in New York City have taken care to make the experience of visitors to their site less intense and generally more paratelic than one to the Holocaust museum. Clinical psychologist Billie Pivnick advised the designers of the September 11 Museum in New York City (Miller, 2014). She tried to insure that the September 11 Museum is psychologically impactful but not unbearable for visitors: the museum incorporates:

lots of exits so people don't feel claustrophobic and... [has built in] tissue boxes in key places in the museum so people could feel "safely held." To recognize the "shock, surprise, and violence" of the attack, "the exhibits became more vertical and jutting." Near the exit of the museum, visitors can sign a piece of steel "electronically," and as a result, "People can leave their mark on the site. They can turn passive into active, which is one way you help people cope with trauma... [this] brings them back to the present in case the exhibits sort of collapsed time for them and they felt stuck in the past." (Miller, 2014) 


\section{Conclusion}

Having the sort of sensory experiences we prefer can be good in itself, but can also support higher levels of performance on telic-type tasks. Comfort optimization needs to be carefully planned for people in a paratelic state to prevent them from relaxing to the extent that paratelic experiences are not optimized. It is important to recognize, however, that energized sensory experiences can detract from paratelic experiences when through excess, etc., they become unpleasant. As Veitch (2012) reports in her summary of related literature: "Regardless of whether one has chosen the conditions or has the power to alter them, working under preferred conditions can create a state of positive affect that in turn leads to benefits in the form of increased cooperation, reduced competition, improved intellectual performance, and increased creativity." Well-being, in general, is increased when the experiences we are having align with those we prefer at that moment.

Once the telic and paratelic states are added into research designs as moderator variables, a whole new set of possibilities arises for research and, as a result of this research, for the design of spaces. More generally, reversal theory gives us a new way of looking at human creativity and in this paper we have seen how the mechanisms involved in relation to the protective frame might play out in both the creation and the use of works of architecture.

\section{References}

Apter, M.J. (1982). The experience of motivation: The theory of psychological reversals. London: Academic Press.

Apter, M.J. (1991). A structural-phenomenology of play. In J.H. Kerr \& M.J. Apter (Eds.), Adult play: A reversal theory approach. Amsterdam: Swets \& Zeitlinger.

Apter, M.J. (2001). Reversal theory as a set of propositions. In M.J. Apter (Ed.), Motivational styles in everyday life: A guide to reversal theory. Washington, DC: American Psychological Association.

Apter, M.J. (2007). Danger: Our quest for excitement. Oxford: One World Press.

Apter, M.J. (2014). Towards a theory of things: Reversal theory and design. Journal of Motivation, Emotion and Personality, 2, 3-11.

Augustin, S. (2009). Place advantage: Applied psychology for interior architecture. New Jersey: Wiley.

Barker, R. (1968). Ecological psychology: Concepts and methods for studying the environment of human behavior. Stanford University Press: Stanford, CA.

Dazkir, S. \& Read, M. (2012). Furniture forms and their influence on our emotional responses toward interior environments. Environment and Behavior, 44(5), 722-732.

De Vries, M., Holland, R., Chenier, T. Starr, M. \& Piotr Winkelman (2010). Happiness cools the warm glow of fa- miliarity: Psychophysiological evidence that mood modulates the familiarity-affect link. Psychological Science, 21(3), 321-328.

Durão, M.J. (2007). Paper presented at Senses, Brain, and Spaces Workshop. University of Salford, http://www.rgc.salford.ac.uk

Fehrman, K. \& Fehrman, C. (2000). Color: The secret influence. Prentice Hall: New York.

Fell, D. (2010). Wood in the human environment: Restorative properties of wood in the built indoor environment (Doctoral dissertation, University of British Columbia, Vancouver, Canada).

Fokkinga, S. \& Desmet, P. (2014a). Introduction to the design issue, Journal of Motivation, Emotion and Personality, 2, 1-2.

Fokkinga, S. \& Desmet, P. (2014b). Reversal theory from the design perspective. Journal of Motivation, Emotion and Personality, 2, 12-26.

Joye, Y. \& Jan Verpooten, J. (2013). An exploration of the functions of religious monumental architecture from a Darwinian perspective. Review of General Psychology, 17(1), 53-68.

Kellert, S. (2008). Dimensions, elements and attributes of biophilic design. In Stephen Kellert, Judith Heerwagen, \& Martin Mador (Eds.), Biophilic design: The theory, science, and practice of bringing buildings to life. Wiley: New York, 3-19.

Kellert, S. (2012). Birthright: People and nature in the modern world. Yale University Press: New Haven, CT.

Kellert, S., Heerwagen, J. \& Mador, M. (2008). (Eds.). Biophilic design: The theory, science, and practice of bringing buildings to life. Wiley: New York.

Kerr, J.H., \& Apter, M.J. (1991). (Eds.). Adult play: A reversal theory approach. Amsterdam: Swets \& Zeitlinger.

Kerr, J.H., Hayashi, R., Matsumoto, M., \& Miyamoto, N. (2002). The influence of settings and environmental events on metamotivational state and arousal: Further tests of reversal theory on Asian samples. Journal of Environmental Psychology, 22, 361-367.

Kerr, J.H. \& Tacon, P. (1999). Psychological responses to different types of locations and activities. Journal of Environmental Psychology, 18 287-294.

Knez, I. \& Enmarker, I. (1998). Effects of office lighting on mood and cognitive performance and a gender effect in work-related judgment. Environment and Behavior, 30(4), 553-567.

Knoop, M. (2007). In, Barrett, P. \& Barrett, L. (Eds.). Senses, Brain and Spaces Workshop. University of Salford, http://www.rgc.salford.ac.uk

Mahnke, F. (1996). Color, environment and human response: An interdisciplinary understanding of color and its use as a beneficial element in the eesign of the architectural environment. Van Nostrand Reinhold: New York. 
Miller, A. (2014). Memorializing a tragedy. Monitor on Psychology, 45(8), 28-31.

Norman, D. (2004). Emotional design. Basic Books: New York.

Russell, J. \& Lanius, U. (1984). Adaptation level and the affective appraisal of environments. Journal of Environmental Psychology, 4, 119-135.

Russell, J. \& Mehrabian, A. (1975). Task, setting, and personality variables affecting the desire to work. Journal of Applied Psychology, 60, 518-520.

Russell, J. \& Mehrabian, A. (1978). Environmental task, and temperamental effects on work performance. Humanitas, 14, 75-95.

Russell, J. \& Snodgrass, J. (1987). Emotion and the environment. In D. Stokols \& I. Altman (Eds.), Handbook of environmental psychology, vol. 1 (pp. 245-280). John Wiley \& Sons: New York.

Schweitzer, M., Gilpin, L., \& Susan Frampton. S. (2004). Healing spaces: Elements of environmental design that make an impact on health. The Journal of Alternative and Complementary Medicine, 10(supplement 1), s71-s83.

Valdez, P. \& Mehrabian, A. (1994). Effects of color on emotions. Journal of Experimental Psychology: General, 123(4), 394-409.

Veitch. J. (2012). Work environments. In Susan Clayton (Ed.), The Oxford handbook of environmental and conservation psychology (pp. 248-275). Oxford University Press: New York.

Walters, J. Apter, M.J. \& Svebak, S. (1982). Color preference, arousal, and the theory of psychological reversals. Motivation and Emotion, 6(3), 193-215.

Wicker, A. (2012). Perspectives on behavior settings: With illustrations from Allison's ethnography of a Japanese hostess club. Environment and Behavior, 44(4), 474-492.

Yerkes, R.M.\& Dodson, J.D. (1908). The relation of strength of stimulus to rapidity of habit-formation. Journal of Comparative Neurological Psychology, 18, 459-482. 